\title{
The Effect of Honey as a Treatment for Oral Ulcerative Lesions: A Systematic Review
}

\author{
Maddison Hunter ${ }^{1,2}$, Jane Kellett ${ }^{1}$, Nathan M. D’Cunha ${ }^{1}$, Kellie Toohey ${ }^{1,2,3}$, \\ Andrew McKune ${ }^{1,3,4}$ and Nenad Naumovski ${ }^{1 *}$
}

\begin{abstract}
${ }^{1}$ Faculty of Health, University of Canberra, Canberra, Australia; ${ }^{2}$ Prehabilitation, Cancer, Exercise and Survivorship (PACES) Research Group, University of Canberra, Canberra, Australia; ${ }^{3}$ Research Institute for Sport and Exercise (UCRISE), Faculty of Health, University of Canberra, Canberra ACT, Australia; ${ }^{4}$ Discipline of Biokinetics, Exercise and Leisure Sciences, School of Health Sciences, University of KwaZulu-Natal, Durban, KwaZulu-Natal, South Africa
\end{abstract}

\begin{abstract}
Background and objectives: A healthy oral environment features a rapid turnover rate of epithelium cells capable of regeneration and repair, with the oral epithelium contributing as a physical barrier and immune defense. However, the oral cavity can be subjected to unique damage, such as ulcerations. Honey is reported as a therapeutic agent for wound healing, due to its antioxidant, antibacterial and anti-inflammatory properties.

Methods: A systematic review was performed following the PRISMA 2015 Guidelines, to assess the efficacy and safety of the therapeutic use of honey in the oral cavity. Four electronic databases were searched (PubMed, Cochrane Library, Scopus, and Web of Science) for randomized controlled trials examining the effect of honey on oral cavity conditions.

Results: In total, 2,832 records were identified, and after applying exclusion criteria, 13 studies were included. Honey was applied topically throughout, for chemotherapy or radiotherapy-induced oral mucositis $(n=11)$, dental wounds $(n=1)$, and recurrent aphthous stomatitis $(n=1)$, all of which are ulcerations with different pathologies. In the majority of studies (12/13), honey reduced the severity and/or duration of the condition compared with control groups (all $p<0.05$ ). However, a group treated with Manuka honey $(n=1)$ experienced adverse effects and considerable participant attrition.

Conclusions: Honey is an effective treatment for a range of oral ulcerative conditions. Future research should focus on compositional analysis of honeys to determine those with optimal beneficial properties, and whether Manuka honey is safe to use in the oral cavity.
\end{abstract}

Introduction

The oral cavity consists of a range of structures, including the

Keywords: Honey; Oral health; Ulcer; Oral cavity.

Abbreviations: ROS, reactive oxygen species; PRISMA, preferred reporting items for systematic reviews and meta-analyses; PICOS, population, intervention, comparator, outcomes and setting; OM, oral mucositis; RAS, recurrent aphthous stomatitis; MGO, methylglyoxal; GSH, glutathione.

Received: November 01, 2019; Revised: December 10, 2019; Accepted: December 16, 2019

*Correspondence to: Nenad Naumovski, Faculty of Health, University of Canberra, P.O. Box 5018, Bruce, ACT 2617, Australia. Tel: +61-(0)2-6206-8719; E-mail: nenad. naumovski@canberra.edu.au

How to cite this article: Hunter M, Kellett J, D'Cunha NM, Toohey K, McKune A, Naumovski N. The Effect of Honey as a Treatment for Oral Ulcerative Lesions: A Systematic Review. Exploratory Research and Hypothesis in Medicine 2020;5(1):27-37. doi: 10.14218/ERHM.2019.00029. teeth, tongue, gingiva, and hard and soft palettes, with the latter being composed of stratified squamous cells. ${ }^{1}$ A healthy oral environment is characterized by a rapid cellular turnover rate, with the soft and hard palettes averaging between 14 and 24 days, respectively. ${ }^{2}$ One of the main purposes of the rapid turnover of the oral epithelium is as a defense mechanism, reducing the rate of pathogenic microbial colonization by removing the region they bind to. ${ }^{2}$ It also serves multiple additional functions, such as a physical barrier and immune defense to potential pathogens and toxins and in providing an environment that is optimal for the established healthy microbiome.

The turnover of the mucosal cells in the oral cavity is dependent on a balance between cell differentiation and desquamation, which primarily acts as a pathogen defense mechanism. ${ }^{3}$ However, this can become unbalanced and lead to the development of several conditions, including hyperplasia and dysplasia, and a reduced rate of proliferation, which can lead to the development of ulcera- 
tions. ${ }^{2}$ Additionally, the oral mucosa is subject to masticatory and abrasive damage, with this oral epithelium disruption having the possibility of leading to the development of oral diseases ${ }^{1}$; some of these common oral conditions include dental caries, denture wounds, and ulcerations. ${ }^{4}$

The treatment of adverse health conditions in the oral cavity is focused on therapies that simultaneously eradicate pathogenic microbial growth, stimulate the wound healing process, and reduce the sensation of pain. One possible treatment option that fulfils these criteria is honey, which has been demonstrated to be effective in the treatment of a range of wounds, such as burns and ulcers. ${ }^{5}$ These potentially beneficial effects can be attributed to its antioxidant, antibacterial, and anti-inflammatory properties. ${ }^{5-7}$

The composition of honey, in particular the exact proportions of each of its constituents, is dependent on the floral source of the pollen used to produce the honey. ${ }^{8}$ Overall, honey is a mixture of several different categories of compounds, including polyphenols, ascorbic acid, carotenoids, organic acids, enzymes and other proteins, ${ }^{9}$ all of which contribute to honey being a viable nutritional source of antioxidants..$^{10}$ Honey displays strong antioxidant activity and has the ability to reduce the effects of oxidative reactions, which produce free radicals and reactive oxygen species (ROS) ${ }^{8}$ In addition, honey is also reported to exhibit strong antibacterial effects, which can be attributed to its physicochemical properties, such as high osmolarity (due to high sugar levels) ${ }^{5}$ and relatively low $\mathrm{pH}^{11}$ due to the presence of several organic acids. Additionally, activation of glucose oxidase during the dilution of honey causes the production of hydrogen peroxide from glucose metabolism, providing an undesirable environment for growth and proliferation of bacterial cells ${ }^{12}$ and potentially mediating the wound healing process. ${ }^{13}$

The concomitant effects of the antioxidant and antibacterial properties of honey also contribute to its anti-inflammatory effects of reducing excessive inflammation, possibly resulting in a wound-healing effect. ${ }^{6}$ As the presence of ROS has been determined to lead to the production of inflammation, ${ }^{14}$ the antioxidant activity of honey can also contribute to a reduction of an excessive inflammatory response. ${ }^{7}$ Moreover, honey's capability to prevent the development of bacterial infections (through providing an environment that cannot support bacterial growth and proliferation) can further assist with the inhibition of inflammation. ${ }^{5}$ Furthermore, the application of honey to a wound has been demonstrated to stimulate the production and release of pro-inflammatory cytokines that assist in the wound healing process, such as interleukin-1 and tumor necrosis factor-alpha. ${ }^{5}$ The topical application of honey to various injured tissues has also been shown to stimulate wound repair through the stimulation of growth of epithelial cells, reduction of edema, and wound debridement. ${ }^{6,7}$

It is well established that an individual's oral health impacts their quality of life. ${ }^{4,15,16}$ Poor oral health is shown to affect physical and psychological wellbeing through condition-related reductions in functionality, including the inability to consume adequate nutrition and communicate, undesirable effects on physical appearance, in addition to causing pain. ${ }^{15}$ These conditions, particularly those related to the soft and hard palette such as ulcerations, have been reported as treated using various types of honey in several different clinical populations. ${ }^{17-19}$ These collective data suggest honey as a reliable food product, being both financially affordable and readily available, that could be used in the treatment of inflammatory and microbial damage of the epithelial lining of the oral cavity. Therefore, this systematic review aimed to assess the efficacy of honey as a therapeutic agent used for the treatment of epithelial damage due to the inflammatory and microbial response in the oral cavity. Additionally, this review will aim to evaluate the safety of honey in different clinical populations.

\section{Methods}

This systematic review has been completed in accordance with the Preferred Reporting Items for Systematic Reviews and MetaAnalyses (PRISMA) $2015^{20}$ Guidelines and was registered in the International Prospective Register of Systematic Reviews (PROSPERO Number: CRD42019128480). The systematic review was completed following the Population, Intervention, Comparator, Outcomes and Setting (PICOS) approach as follows:

Population: Human populations with an existing oral health condition, or at risk of developing an oral health condition.

Intervention: Randomized controlled trials utilizing topicallyapplied undiluted honey from any floral source as a treatment or preventative agent without the concurrent use of other treatments. Only trials containing sample sizes over 12 participants were included; however, there was no time limit placed on study duration.

Comparator: Human comparators being treated with a placebo, control or routine standard oral care.

Outcomes: Effects of honey treatment on severity of oral health conditions, improvements in healing time, and any adverse effects to participants caused by the use of honey during and following the intervention protocol.

Setting: Any.

\section{Search strategy}

The following four electronic databases were searched; Scopus, PubMed, Cochrane Library and Web of Science, for manuscripts published since journal inception until June 2019. Furthermore, searches were also conducted to identify any new publications prior to submission of this manuscript (end of October 2019). The searches were limited to studies that were conducted on humans, were randomized controlled trials, and were published in peer-reviewed journals in the English language. The reference lists of included articles were also searched to locate any additional articles relating to the topic. The search terminology used included "honey*" and "oral", "mouth", "intraoral", "cavity", "bucc*", "tooth", "orthodont*", "saliv*", and "disease", "condition", "lesion", "wound", "carie*”, "plaque", "ulcer", "bacter*", "micro", "count", with the terms AND and OR between every term.

\section{Selection criteria}

Studies were included if they were original research, randomized controlled trials published in English and in peer-reviewed journals. Articles were only considered if they utilized undiluted honey without any other interventions as a treatment against a pre-existing condition of the mouth or as a preventative agent in a group more susceptible to developing an oral condition. Furthermore, articles were required to have a control or placebo group as a comparator. Any articles that did not meet these criteria were excluded from this review. There were no time limitations posed on the duration of the studies, as oral conditions have different durations and pathological severities. For the purpose of this systematic review, a condition of the mouth was defined as a symptomatic problem unique to the oral cavity, such as ulcers or dental wounds.

\section{Data extraction}

All articles identified in the initial electronic search were imported 
into an EndNote library (vX8.2; Clarivate Analytics, USA) and duplicates removed. We excluded articles that did not investigate honey or oral health conditions. Following this, articles were excluded based on study design, and only if pure undiluted honey was utilized in the absence of any other treatments, with the exception of patient-required analgesics.

Two authors ( $\mathrm{MH}$ and $\mathrm{JK}$ ) independently screened each of the articles based on the selection criteria. The titles were screened to determine relevance, followed by screening of abstracts to determine studies suitable for full-text analysis. Any disagreements regarding the selection criteria were resolved through discussion until consensus was reached or in consultation with a third author $(\mathrm{NN})$. At this stage, the reference lists of the articles considered for full-text review were searched for additional articles. The extraction of relevant data was completed by two authors (MH and JK) working independently in predesigned tables including the information on author name, year of publication, condition assessed, honey application protocol, control or placebo, number of participants, and outcomes.

\section{Assessment of bias}

The risk of bias was independently assessed according to the Cochrane Collaboration's Tool for Assessing Bias, which utilizes the domains of selection bias through random sequence generation and allocation concealment, performance bias through blinding of participants and personnel, detection bias through blinding of outcome assessment; attrition bias through the addressing of any incomplete data, reporting bias through selective reporting, and any other sources of bias. ${ }^{21}$ The risk of bias was assessed independently by two authors (MH and JK), with a third author (NN) introduced in the case of any disagreements, where appropriate.

\section{Data analysis}

Due to the broad inclusion criteria used for this review to include a number of ulcerative lesions, which includes a variety of population groups and intervention protocols, this review consists of considerable heterogeneity. As such, a meta-analysis was not deemed appropriate.

\section{Results}

\section{Study selection}

The initial search of the electronic databases (Fig. $1^{20}$ ) resulted in 2,838 articles, with the secondary searches identifying 1 article. Following removal of articles due to duplicates $(n=$ $1,181)$, study design $(n=115)$, absence of honey investigation $(n=1,416)$ and absence of oral health intervention $(n=80)$, 46 studies were subjected to full text-analysis. Articles were further excluded due to use of diluted honey with or without the use of additional therapies $(n=14)$, unsuitable oral health conditions $(n=12)$ and study design $(n=8)$, with 13 studies fitting the inclusion criteria. ${ }^{22-34} \mathrm{An}$ article investigating the use of honey to assist in the recovery of dental extraction surgery ${ }^{35}$ was considered but it was determined that since this wound is due to surgery, and not a condition unique to the oral cavity, this article was excluded.

\section{Study characteristics}

The characteristics of the included studies are presented in Table $1 .^{22-34}$ The total number of participants recruited in the included studies was 634, with sample sizes ranging from 20 to 106 participants. Of these, 11 studies investigated oral mucositis (OM) in 579 cancer patients, ${ }^{22-32} 1$ investigated 35 participants with denture-induced ulcers, ${ }^{33}$ and 1 investigated 20 participants with recurrent aphthous stomatitis (RAS). ${ }^{34}$ Although the selection criteria of this review was designed to include a broad range of oral health conditions, the intervention was common across studies, with pure, undiluted honey being applied topically to affected areas in the mouths of patients in the treatment groups. The type of honey and their floral sources used in the included studies was largely unspecified ${ }^{26,28,30,33,34}$; however, two studies stated that the honey was sourced from a commercial supermarket. ${ }^{23,27}$ Five of the studies identified the local flora that was used in the production of the honey used,,$^{22,24,29,31,32}$ and one study specified their use of Manuka honey. ${ }^{25}$

In the included articles, participants were instructed to maintain good oral hygiene $(n=6)^{22,24-26,31,32}$ or prescribed the use of antibacterial, antifungal or analgesic solutions $(n=3) .^{22,23,32}$ However, this was not specified in six studies. ${ }^{27-30,33,34}$ For trials investigating $\mathrm{OM}(n=11)$, the studies investigated head and neck cancer patients in adult populations $\mathrm{s}^{24-32}$ and pediatric patients receiving treatment for a range of cancers, including leukemia. ${ }^{22,23}$ Participants with radiotherapy-induced OM were included in six studies, ${ }^{24,25,27-29,31}$ with 1 study having investigated chemotherapy-induced $\mathrm{OM}^{22}$ and 4 having determined the effect of honey on concurrent chemo-radiotherapy-induced OM. ${ }^{23,26,30,32}$ These studies utilized routine oral care, ${ }^{23,24,30,32}$ saline rinses of different concentration ranges $\left(0.9 \%,{ }^{27} 0.09 \%,{ }^{31}\right.$ and unspecified $\left.{ }^{26}\right)$, water, ${ }^{28}$ and a variety of anesthetic and analgesic solutions $(7.5 \%$ benzocaine gel, ${ }^{22} 15 \%$ benzydamine hydrochloride, ${ }^{27}$ lignocaine gel $^{29}$ ) serving as controls, while only one study utilized a placebo gel $^{25}$ and another developed their own mixture. ${ }^{22}$

\section{Interventions}

In a study by Biswal et al.,${ }^{24}$ patients receiving radiotherapy for head and neck cancer were provided with $20 \mathrm{~mL}$ of honey to smear on the inside of their mouths, and instructed to swallow it slowly in order to coat both the oral and pharyngeal mucosa, with application occurring 15 min before and after they received radiotherapy and $6 \mathrm{~h}$ after receiving radiotherapy, for the duration of the treatment cycle. This study design was also replicated by other studies included in this review, ${ }^{27,29-32}$ with Jayalekshmi et al. ${ }^{28}$ and Howlader et al. ${ }^{26}$ with slight modifications, namely use of 15 $\mathrm{mL}$ of honey ${ }^{28}$ and consumption of additional honey to potentially contribute to serum antioxidant levels. ${ }^{26}$

The remaining studies investigating OM utilized study designs similar to those above, topically coating the oral cavity with honey; however, the amounts of honey used and timing of application were different. In trials by Al Jaouni et al. ${ }^{23}$ and Hawley et $a l .{ }^{25}$ durations were similar of treatments used to Biswal et al. ${ }^{24}$ and participants were followed throughout the duration of their oncology treatments. In addition, Hawley et al. ${ }^{25}$ maintained the intervention for 7 days following final radiotherapy treatment, with all studies applying the honey intervention four times daily. The volumes of honey and subsequent saline rinse were not specified by Al Jaouni et al. ${ }^{23}$ but Hawley et al. ${ }^{25}$ specified the prescription of $5 \mathrm{~mL}$ of applied honey followed by a fluoride rinse. Abdulrhman 


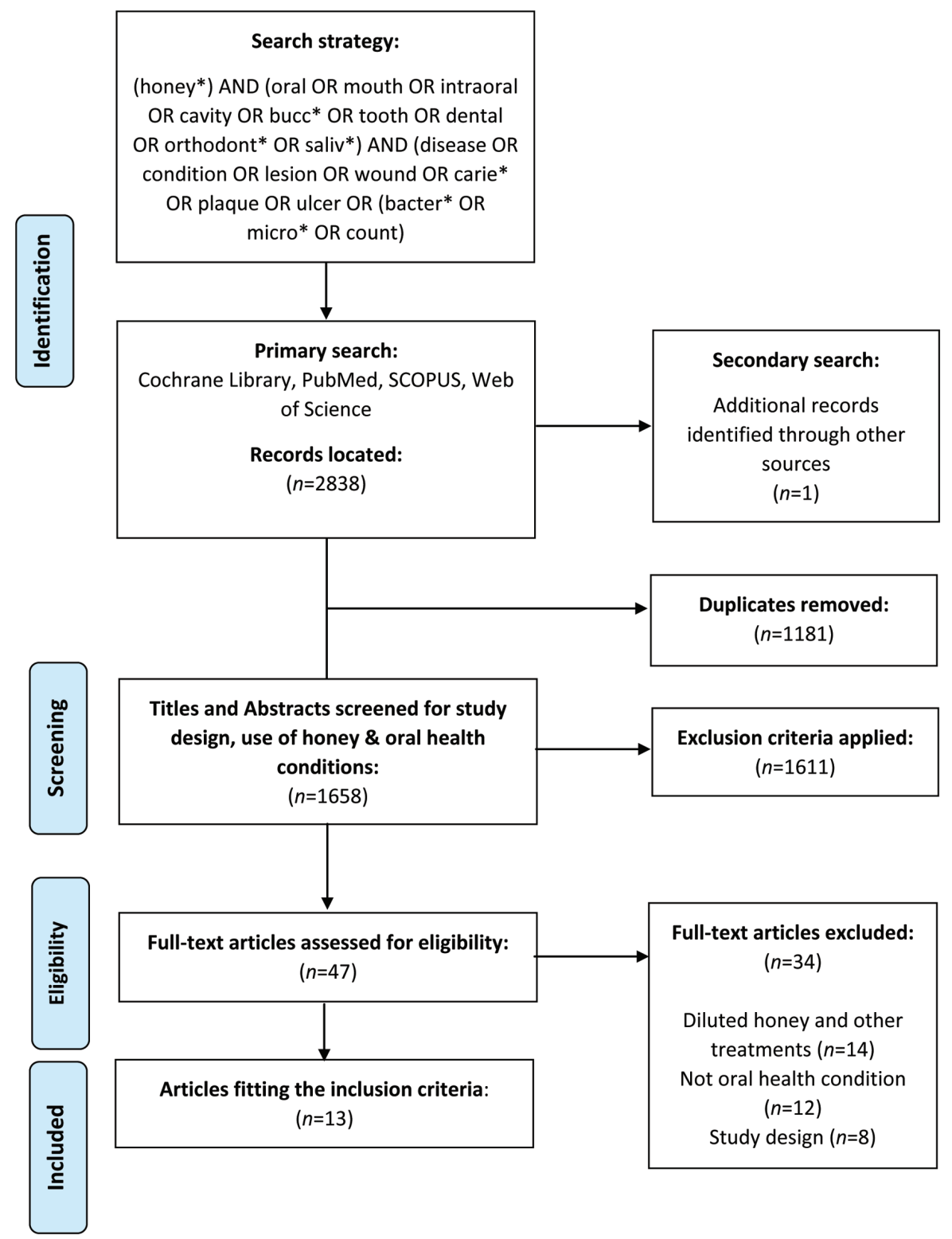

Fig. 1. Search strategy and article selection process according to the Preferred Reporting Items for Systematic Reviews and Meta-Analyses (PRISMA) Guidelines. $^{20}$

et al. ${ }^{22}$ included participants with established OM using undiluted honey $(0.5 \mathrm{~g} / \mathrm{kg})$ and having two control groups (receiving a custom-made mixture (placebo) or an analgesic control). All groups applied their mixtures three times daily for 10 days or until healing of ulceration.

The study by Ceylan et al. ${ }^{33}$ investigated the use of honey in the treatment of traumatic ulcers that were caused by newly fitted dentures in 35 healthy, previously edentulous participants. Of these, 25 participants were provided with honey and instructed to smear directly onto the ulcers three times daily for 3 days following denture fitting. The control group $(n=10)$ were provided with a saline rinse to use via the same protocol as the treatment group. The ulcer areas were measured in all participants using planimetry on the first and third day following initial denture fitting.

The study by Halim et $a l .{ }^{34}$ investigated the effectiveness of honey in the treatment of RAS in healthy adults. Following an initial assessment, participants in the treatment group $(n=10)$ were given honey and control groups $(n=10)$ were given salicylate gel, a standard prescribed treatment for RAS, to apply to their ulcers 3 times daily for 5 days. Ulcer size was determined using calipers, and a visual analog score was used to assess pain before and after the intervention.

\section{Study results}

The majority of studies investigating the use of honey in the treatment of OM in an oncology population were successful in alleviating negative symptoms, including reducing the severity or duration of the condition (all $p<0.05$ ) (Table 1). ${ }^{22-24,26-29,31,32}$ In these stud- 


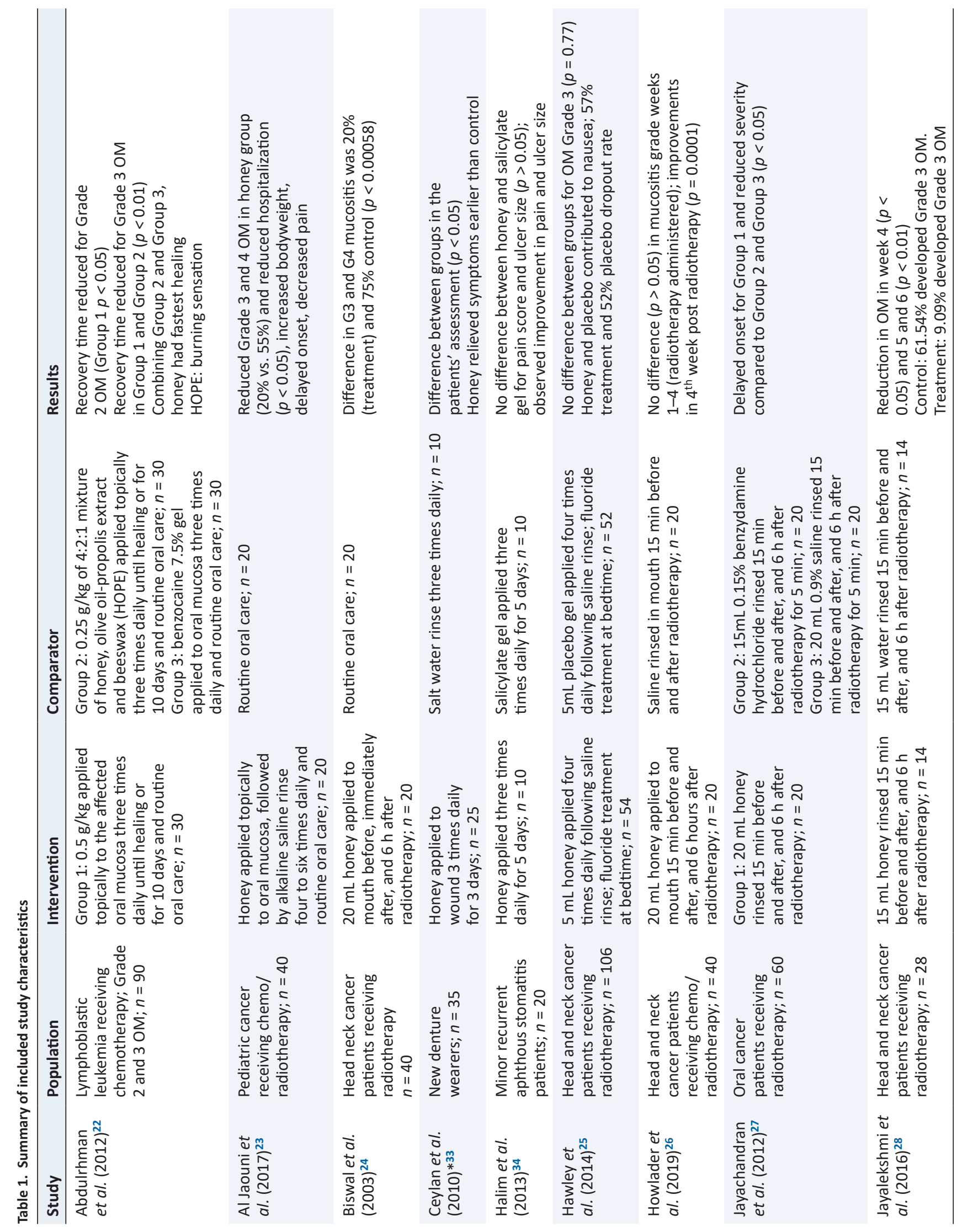




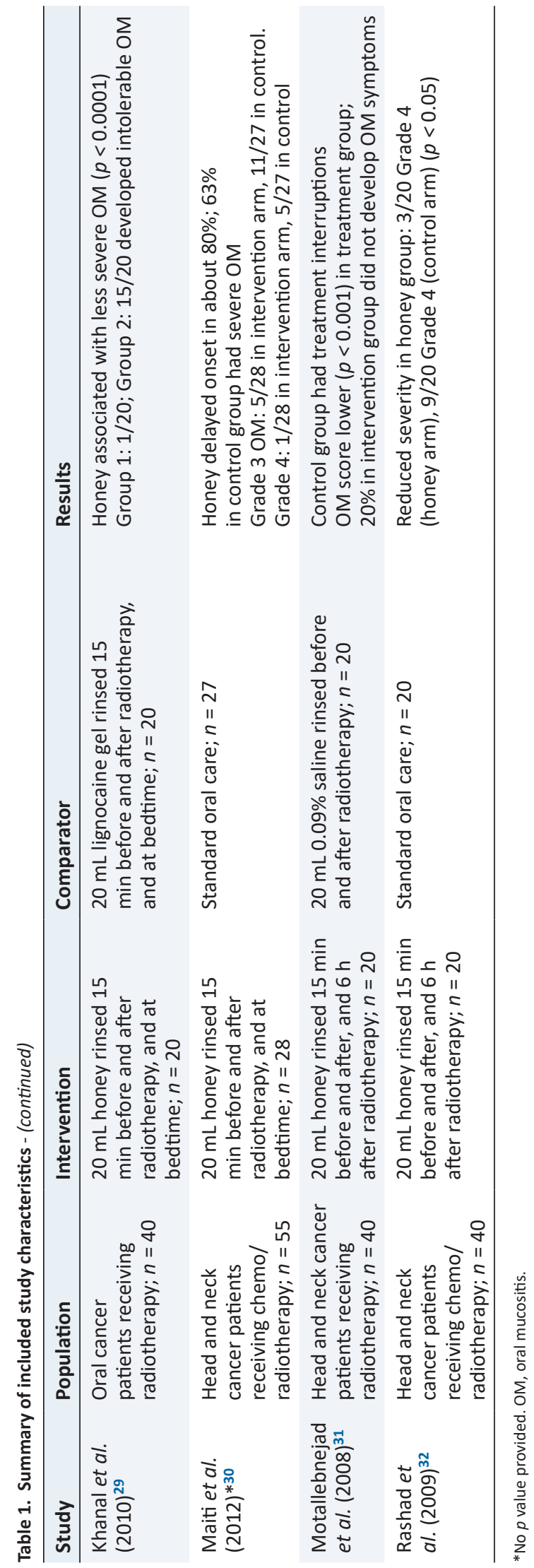

ies, OM was assessed using a grading scale, with Grade 1 representing less severe cases and Grade 4 being the most severe. In these studies, the onset of OM was delayed in the honey-treatment group compared to the control group, and fewer participants in the honey group of each study developed higher gradings of the condition. One OM study did not report any significant changes from pre- to post-treatment nor any difference between the two groups (treatment and control); however, there was a self-reported observed difference in the OM. ${ }^{30}$ All of these studies utilized standard honey from no particular floral source, with no adverse reactions reported throughout the duration of the honey intervention, excepting one study ${ }^{31}$ that reported the exclusion of participants who reported a stinging sensation, though the number of exclusions and effect on the study was not disclosed. A single study by Hawley et $a l .{ }^{25}$ utilized Manuka honey and reported no significant difference in the severity or duration of OM between the honey and placebo groups $(p=0.77)$; however, this study experienced considerable dropout rate (57\% in the honey group, $52 \%$ in the placebo group), mainly attributed to the sensation of nausea caused by the application of the honey.

It was determined that honey significantly ( $p$ value unreported) relieved the symptoms of the denture-induced ulcers earlier than in the control group, and (as assessed by a visual analogue score) reduced pain, with no adverse effects. ${ }^{33}$ Finally, while no participants with RAS reported adverse effects, no significant difference was found between the honey group and control group of a currently utilized treatment for ulcer size $(p=0.879)$ and pain $(p=0.514)$; however, self-reported improvements were observed. ${ }^{34}$

\section{Risk of bias in included studies}

The risk of bias was assessed according to the Cochrane Risk of Bias Tool and is outlined in Table $2 .{ }^{22-34}$ The risk of selection bias through allocation concealment was high for the included studies, which was due to placebo products being utilized by $2 / 13$ studies. ${ }^{22,25}$ Additionally, Jayalekshmi et al. ${ }^{28}$ reported the use of similar bottles for both their treatment and control groups, resulting in a low risk of selection bias. Similarly, the performance bias was high or unclear, which was attributed to the common utilization of controls rather than a placebo. The detection bias for the included studies was relatively unclear but there was an overall low risk of attrition and reporting bias (Table 2). No other bias was detected.

\section{Discussion}

\section{Effectiveness of honey}

The primary aim of this review was to determine the effectiveness of topically-applied honey in the treatment of a range of oral health conditions. Of the 13 studies included in this review, 10 reported statistically significant reductions in the severity of the oral health condition compared to a control group, including 9 studies investigating $\mathrm{OM}^{22-24,26-29,31,32}$ and 1 investigating denture wounds. ${ }^{33}$ While one study did not report any significance ${ }^{30}$ and another reported no significant difference in the improvement of RAS, ${ }^{34}$ the oral condition was improved in both studies, demonstrating that honey was just as effective as the standard prescribed treatments for these conditions. Only one study, by Hawley et al. ${ }^{25}$ did not report any improvements in oral condition.

It has been proposed that honey possesses wound healing abili- 


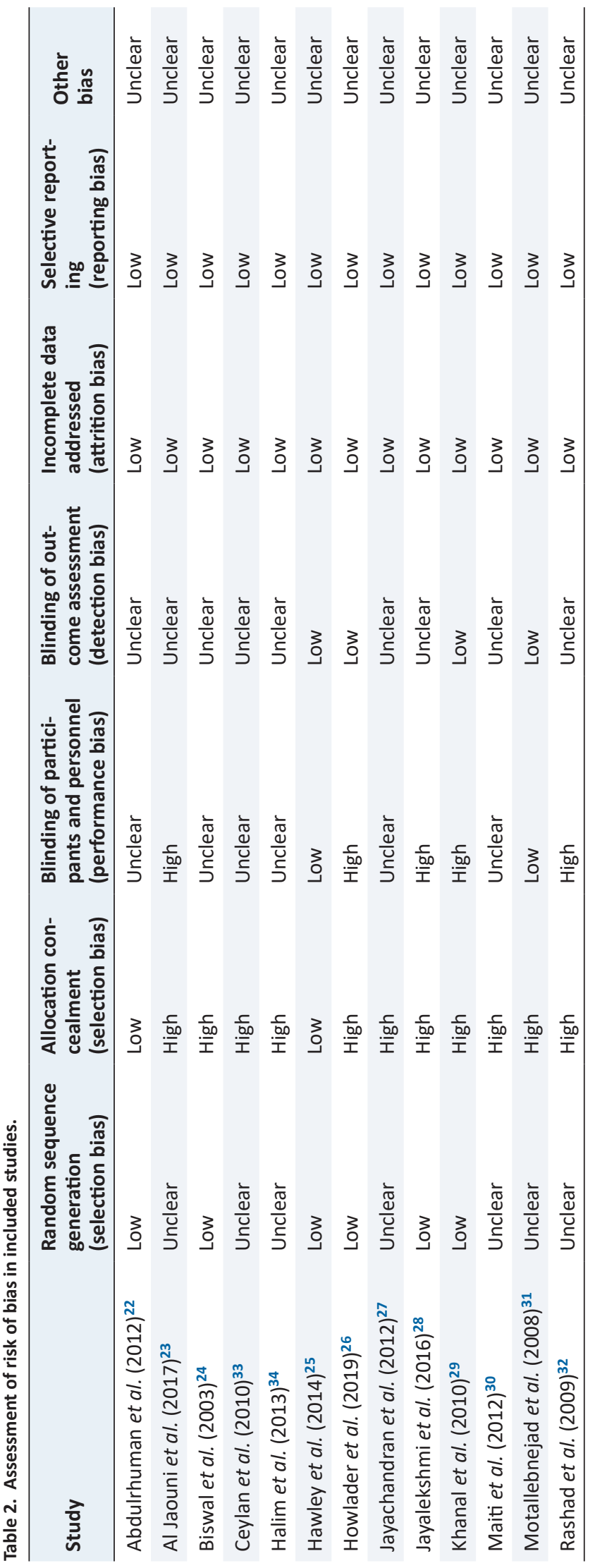

ties due to its antioxidant and antibacterial properties, ${ }^{5-7}$ which includes the ability to heal ulcerations. All conditions included in this review culminated in the development of an oral ulcer, which had formed due to the loss and necrosis of epithelial tissue and resulting in breaks in the skin or mucous membrane ${ }^{36}$; each condition formed due to different pathologies. Oral mucositis is a condition that develops as a toxicity during oncology treatments (chemotherapy and radiotherapy) and is characterized by inflammation, erythema, microbial colonization, and ulceration. ${ }^{37}$ Similarly, RAS is a condition of unknown cause that affects the lining of the mouth, causing erythema, burning and ulceration, ${ }^{34}$ and denture-induced ulcers are caused by the introduction of ill-fitting dentures causing abrasive damage to the lining of the mouth, resulting in ulcerative lesions. ${ }^{33}$

The antibacterial and antioxidant properties of honey contribute to honey's wound-healing abilities. In the case of OM, the initiation phase is characterized by the production of ROS, ${ }^{37}$ which could potentially cause further damage to the affected areas. A study investigating participants with RAS determined that those with the condition had significantly lower salivary antioxidant levels compared to healthy controls,${ }^{38}$ demonstrating that the ulcerations potentially produced ROS, causing a reduction in the antioxidant compounds present in the mouth. This highlights a possible mechanism for the effectiveness of honey as a treatment for the ulcerations discussed in this review. Potentially, the presence of the antioxidant compounds provided by the topically-applied honey can reduce the effects of ROS activity during the development of ulcerations. Furthermore, the consumption of honey increases total systemic plasma antioxidant levels, and swallowing of the topically-applied honey may contribute to longer lasting antioxidant effects, ${ }^{10}$ further contributing to the wound healing process.

\section{Adverse effects}

The secondary aim of this systematic review was to determine any adverse effects during the topically-applied honey interventions. Only one of the included studies reported an adverse effect due to the honey intervention. ${ }^{25}$ Namely, Hawley et al. ${ }^{25}$ reported considerable dropouts due to nausea, which could be attributed to the treatments being associated with radiotherapy; however, it was reported that the Manuka honey that was used worsened nausea symptoms and encouraged a retching motion. A study by Bardy et al. ${ }^{39}$ also used Manuka honey and reported a low compliance rate due to the honey being difficult to use, along with an undesirable taste and texture. A study by Parsons et al. ${ }^{40}$ initially had to be discontinued due to the Manuka honey not being well-tolerated by participants, and in that study all participants withdrew due to a perceived stinging pain and nausea following application. Interestingly, Abdulrhman et al. ${ }^{22}$ reported no adverse side effects in the honey intervention arm of their study, which used honey from a different floral source to Manuka honey; however, in their comparative group using their own formulation, discomfort was reported, which was proposed to be due to the addition of propolis. The studies investigating denture wounds ${ }^{33}$ and RAS $^{34}$ did not report any adverse effects. This further supports evidence that the application of honey to the inside of the oral cavity is effective in reducing the pathogenic bacteria that cause dental caries, and has a positive effect on plaque levels and gingivitis. ${ }^{17,41,42}$ These findings demonstrate that the use of honey in future studies is safe for the dental health of participants. It was also proposed that similar trials comparing two intervention groups of Manuka and conventional honey should be completed ${ }^{18}$; however, given 
the evidence of the adverse effects of Manuka honey, this may not be feasible.

\section{Issues with use and development of placebo}

The majority of the studies included in this review $(n=12)$ utilized a control or 'standard' prescribed care in the place of a placebo. Due to the nature and progression of oral health conditions included in this review, particularly $\mathrm{OM}$ in oncology patients, it would be unethical to provide no treatment to the control group, resulting in most studies in this review receiving standard care or a commonly-used treatment. This is also partially reflected by the reported bias (Table 2), where many studies were graded as having a "high risk" of selection and performance bias, with the exception of the study by Jayalekshmi et al. ${ }^{28}$ who utilized similar packaging for their honey treatment and control.

If a placebo were to be used in future studies to assess the effectiveness of honey as a topical treatment, it would be best practice to use products which share certain physicochemical properties with honey to support undetectability by participants, such as its properties of osmolarity and acidity. ${ }^{31}$ The use of such a placebo could have confounding effects, as the physicochemical properties of honey, including its osmolarity, contribute to honeys woundhealing abilities. ${ }^{19}$ One such example is brought forth by the study by Bardy et al., ${ }^{39}$ which had been excluded (during full-text review) from our review due to not having used undiluted honey. In that study, golden syrup which has a similar appearance and texture to honey, was assigned as a placebo alongside a Manuka honey treatment for OM. While there was no significant difference observed in the severity or duration of the OM between the treatment and control groups $(p>0.05)$, the pathogenic bacteria present in both groups was consistent with baseline throughout the study period, demonstrating that both honey and the placebo controlled the bacterial growth. This further supports the notion of the importance of osmolarity action (shared by the honey and golden syrup) on the antibacterial activity of honey.

The only study in this review that utilized a placebo was completed by Hawley et al. ${ }^{25}$ where the product was developed to look like honey. Similar to the study by Bardy et al.,${ }^{39}$ this study did not result in a significant reduction in the severity or duration of the OM experienced by participants; however, this could be attributed to the possible non-suitability of Manuka honey for this type of treatment, rather than the activity of the placebo. Additionally, a study by Abdulrhman et al. ${ }^{22}$ developed a honey-containing product, which they assessed as a treatment for OM alongside a treatment group that received pure, undiluted honey, in addition to the use of a standard care control (Table 1).

It is evident that the utilization of a placebo in studies of this nature is not practical, and so further care should be taken in future studies to ensure that both participants and researchers are blinded to the allocation of honey or control. This could potentially be achieved through disclosing that the intervention is designed to improve the oral health condition, while not specifying that honey is the treatment.

\section{Compositional properties of used honeys}

It is well established that several honey properties can contribute to its wound healing effects, including a combination of antioxidant, physicochemical and antimicrobial properties. However, the optimal levels of compounds or categories of compounds, including their interaction with each other, that can contribute to the ob- served beneficial health effects of honey, are largely unexplored. Four of the included studies, all investigating OM, completed in vitro analysis of the potential wound healing properties, including $\mathrm{pH},{ }^{22,24,31,32}$ moisture content, ${ }^{22,24,31,32}$ phytochemical profile, ${ }^{24,32}$ and microbiological analysis. ${ }^{24,31}$ However, none of the studies utilized these results in their discussion on the effectiveness of honey as a treatment. A trial of this nature should be completed to determine the individual in vitro wound healing properties of different types of honey, so that recommendations can be offered to participants experiencing these oral health conditions about what type of honey should be used to provide optimal benefits. Due to the overall lack of analysis of the honey utilized in these trials, it is difficult to draw conclusions about the best type/s of honey and the properties that are most appropriate for use in the treatment of these oral conditions.

\section{Limitations}

The inclusion criteria for this review evaluated only articles that utilized pure, undiluted honey as a treatment, as the concurrent utilization of other compounds or medications has the possibility to contribute to any potential beneficial effect provided by just the honey in isolation. As a result, studies that utilized honey and no other treatments were included, though the use of standard care was allowed. It would be unethical to request that patients discontinue their normal oral care routines for the duration of an intervention, due to the potential impact on their dental health. Additionally, the conditions investigated in this review can cause considerable pain, which could lead to the requirement of the additional analgesics. This may provide a reduction in the effectiveness of the honey; however, the use of honey in isolation to routine oral care in interventions such as the ones included in this review is impractical.

\section{Future research proposals}

The adverse effects caused by Manuka honey highlighted in this review should be taken into consideration of future research for oral health conditions. Manuka honey contains relatively high levels of methylglyoxal (MGO), which has been identified to be unique to this type of honey and directly contributes to Manuka honey's antibacterial abilities. ${ }^{43,44}$ This compound also occurs in humans as a by-product to metabolic processes, such as glycolysis ${ }^{45}$; however, it has been shown to exhibit potentially toxic side effects, including the ability to modify DNA and other macromolecules. ${ }^{46}$ Additionally, in healthy human cells, the tripeptide glutathione (GSH) is capable of suppressing the activity of $\mathrm{MGO}^{47}$; however, once a cell becomes damaged, such as during the oral conditions discussed in this review, there is a reduction in the expression of GSH. ${ }^{48}$ This can result in further damage, with GSH depletion leading to MGO accumulation. ${ }^{49}$ This is depicted in Figure 2, where, in a healthy oral cavity, GSH is able to suppress the activity of MGO, thereby preventing any cellular modifications. Although, when Manuka honey is applied to the damaged oral cavity, the reduced levels of GSH allow the levels of MGO to increase, and to potentially cause further cellular damage.

As previously discussed, one study included in this review reported considerable adverse effects following the topical application of Manuka honey. ${ }^{25}$ Studies of similar interventions also reported adverse effects, ${ }^{39,40}$ which could potentially be due to the proposed interactions between MGO and reduced levels of GSH. These adverse reactions were not observed in studies utilizing hon- 


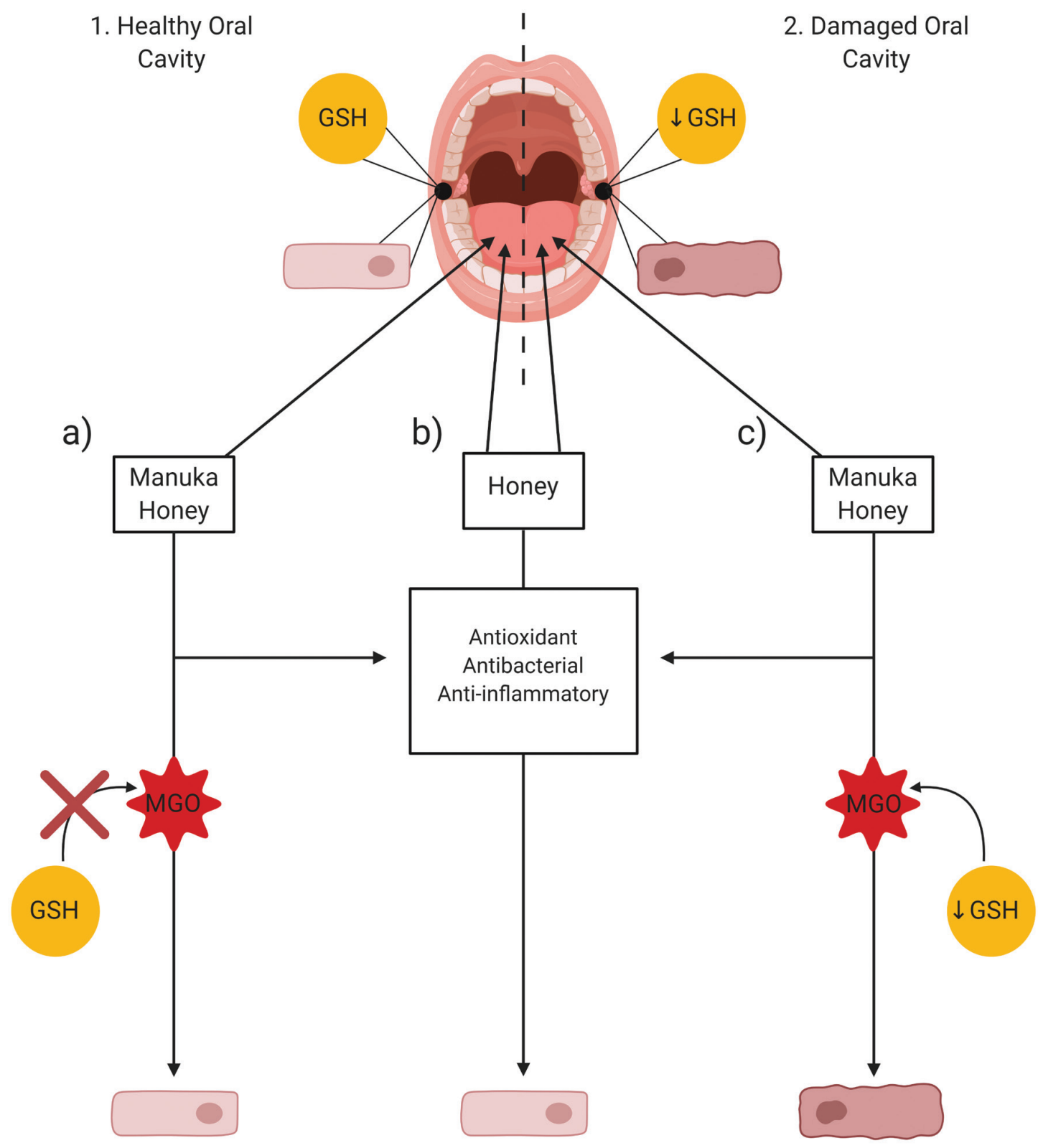

Fig. 2. Effect of honey on the oral cavity. (a) GSH present in the healthy oral cavity suppresses the activity of MGO from Manuka honey. (b) The use of honey does not cause adverse effects in a healthy oral cavity, and is effective in treating a damaged oral cavity. (c) Reduced levels of GSH in the damaged oral cavity do not suppress the activity of MGO from Manuka honey, with the MGO potentially causing further damage, and Manuka honey not being an effective treatment. GSH, tripeptide glutathione; MGO, methylglyoxal.

ey from other floral sources, with the topical application of honey to a damaged oral cavity being demonstrated to result in improvements to the oral condition (Fig. 2).

While Manuka honeys have demonstrated substantial potential wound healing abilities in vitro and in vivo, ${ }^{11,44,50}$ there is currently a lack of relevant clinical trials utilizing this product for wound healing treatments. Due to its compositional properties, it should be considered that Manuka honey be utilized in clinical trials for site-specific topical application in wound sites other than the oral cavity, such as a laceration or burn on the skin to test for effectiveness and tolerability. Similarly, further trials in the treatment of oral conditions or investigating alternative application sites could be completed to determine optimal levels of MGO that can be tolerated.

\section{Conclusion}

The use of honey as a therapeutic treatment of oral conditions has been identified to be effective in reducing the duration and severity of conditions in comparison to standard treatments and controls. Specifically, it has been demonstrated that honey is effective against a range of oral ulcers with multiple pathologies and, in particular, in the treatment of oral mucositis. Any adverse reactions 
that were reported throughout the duration of the included studies could be attributed to the type of honey used and were not due to the type of oral health condition, demonstrating that the topical application of honey, other than Manuka honey, is relatively safe for use across a range of oral health conditions. The use of honey for the treatment of oral mucositis should be considered for further research; however, control over honey composition should be taken into consideration, particularly phytochemical composition. The use of Manuka honey in the treatment of oral cavity conditions should be carefully considered before use, with future investigations required to determine its safety.

\section{Acknowledgments}

None

\section{Funding}

M. Hunter is supported by an Australian Government Research Training Program Scholarship. N. M. D'Cunha is supported by a Dementia Australia Research Foundation PhD Scholarship. All other authors have no sources of funding to declare.

\section{Conflict of interest}

All authors declare no conflict of interests.

\section{Author contributions}

Study concept and design (MH, NN); data acquisition (MH, JK); data analysis $(\mathrm{MH}, \mathrm{JK}, \mathrm{NN})$; drafting of the manuscript $(\mathrm{MH})$; critical revision of the manuscript $(\mathrm{MH}, \mathrm{JK}, \mathrm{NMD}, \mathrm{KT}, \mathrm{AM}, \mathrm{NN})$; administrative, technical, and study supervision (NN).

\section{References}

[1] Wang SS, Tang YL, Pang X, Zheng M, Tang YJ, Liang XH. The maintenance of an oral epithelial barrier. Life Sci 2019;227:129-136. doi:10.1016/j.lfs.2019.04.029.

[2] Qin R, Steel A, Fazel N. Oral mucosa biology and salivary biomarkers. Clin Dermatol 2017;35(5):477-483. doi:10.1016/j.clindermatol.2017.06.005.

[3] Squier CA, Kremer MJ. Biology of oral mucosa and esophagus. J Natl Cancer Inst Monogr 2001;2001(29):7-15. doi:10.1093/oxfordjournals.jncimonographs.a003443.

[4] Haag DG, Peres KG, Balasubramanian M, Brennan DS. Oral conditions and health-related quality of life: a systematic review. J Dent Res 2017;96(8):864-874. doi:10.1177/0022034517709737.

[5] Molan PC, Rhodes T. Honey: a biologic wound dressing. Wounds 2015;27(6):141-151.

[6] Hadagali MD, Chua LS. The anti-inflammatory and wound healing properties of honey. Eur Food Res Technol 2014;239(6):1003-1014. doi:10.1007/s00217-014-2297-6.

[7] Yaghoobi R, Kazerouni A, Kazerouni O. Evidence for clinical use of honey in wound healing as an anti-bacterial, anti-inflammatory antioxidant and anti-viral agent: A review. Jundishapur J Nat Pharm Prod 2013;8(3):100-104. doi:10.17795/jjnpp-9487.

[8] Liu JR, Ye YL, Lin TY, Wang YW, Peng CC. Effect of floral sources on the antioxidant, antimicrobial, and anti-inflammatory activities of honeys in Taiwan. Food Chem 2013;139(1-4):938-943. doi:10.1016/j. foodchem.2013.02.015.

[9] Escuredo O, Seijo MC, Salvador J, González-Martín MI. Near infrared spectroscopy for prediction of antioxidant compounds in the honey. Food Chem 2013;141(4):3409-3414. doi:10.1016/j.foodchem.2013.06.066.

[10] Schramm DD, Karim M, Schrader HR, Holt RR, Cardetti M, Keen CL. Honey with high levels of antioxidants can provide protection to healthy human subjects. J Agric Food Chem 2003;51(6):1732-1735. doi:10.1021/jf025928k.

[11] Alvarez-Suarez JM, Giampieri F, Cordero M, Gasparrini M, ForbesHernández TY, Mazzoni L, et al. Activation of AMPK/Nrf2 signalling by Manuka honey protects human dermal fibroblasts against oxidative damage by improving antioxidant response and mitochondrial function promoting wound healing. J Funct Foods 2016;25:38-49. doi:10.1016/j.jff.2016.05.008.

[12] Bang LM, Buntting C, Molan P. The effect of dilution on the rate of hydrogen peroxide production in honey and its implications for wound healing. J Altern Complement Med 2003;9(2):267-273. doi:10.1089/10755530360623383.

[13] Martinotti S, Laforenza U, Patrone M, Moccia F, Ranzato E. Honeymediated wound healing: $\mathrm{H}_{2} \mathrm{O}_{2}$ entry through AQP3 determines extracellular $\mathrm{Ca}^{2+}$ influx. Int J Mol Sci 2019;20(3):764. doi:10.3390/ ijms20030764.

[14] Safi SZ, Batumalaie K, Qvist R, Mohd Yusof K, Ismail IS. Gelam honey attenuates the oxidative stress-induced inflammatory pathways in pancreatic hamster cells. Evid Based Complement Alternat Med 2016;2016:5843615. doi:10.1155/2016/5843615.

[15] Halvari AEM, Halvari H, Deci EL. Dental anxiety, oral health-related quality of life, and general well-being: a self-determination theory perspective. J Appl Soc Psychol 2019;49(5):295-306. doi:10.1111/ jasp.12583.

[16] Barkokebas A, Silva IHM, de Andrade SC, Carvalho AAT, Gueiros LAM, Paiva SM, et al. Impact of oral mucositis on oral-health-related quality of life of patients diagnosed with cancer. J Oral Pathol Med 2015;44(9):746-751. doi:10.1111/jop.12282.

[17] Atwa ALDA, AbuShahba RY, Mostafa M, Hashem MI. Effect of honey in preventing gingivitis and dental caries in patients undergoing orthodontic treatment. Saudi Dent J 2014;26(3):108-114. doi:10.1016/j. sdentj.2014.03.001.

[18] Münstedt K, Momm F, Hübner J. Honey in the management of side effects of radiotherapy- or radio/chemotherapy-induced oral mucositis. a systematic review. Complement Ther Clin Pract 2019;34:145152. doi:10.1016/j.ctcp.2018.11.016.

[19] Al-Waili N, Salom K, Al-Ghamdi AA. Honey for wound healing, ulcers, and burns; data supporting its use in clinical practice. ScientificWorldJournal 2011;11:766-787. doi:10.1100/tsw.2011.78.

[20] Moher D, Liberati A, Tetzlaff J, Altman DG, PRISMA Group. Preferred reporting items for systematic reviews and meta-analyses: the PRISMA statement. PLoS Med 2009;6(7):e1000097. doi:10.1371/journal. pmed.1000097.

[21] Higgins J, Green S (editor). Cochrane Handbook for Systematic Reviews of Interventions 2011. Version 5.1.0 [updated March 2011]. Available from www.handbook.cochrane.org.

[22] Abdulrhman M, Elbarbary NS, Ahmed Amin D, Saeid Ebrahim R. Honey and a mixture of honey, beeswax, and olive oil-propolis extract in treatment of chemotherapy-induced oral mucositis: a randomized controlled pilot study. Pediatr Hematol Oncol 2012;29(3):285-292. d oi:10.3109/08880018.2012.669026.

[23] Al Jaouni SK, Al Muhayawi MS, Hussein A, Elfiki I, Al-Raddadi R, Al Muhayawi SM, et al. Effects of honey on oral mucositis among pediatric cancer patients undergoing chemo/radiotherapy treatment at King Abdulaziz University Hospital in Jeddah, Kingdom of Saudi Arabia. Evid Based Complement Alternat Med 2017;2017:5861024. doi:10.1155/2017/5861024.

[24] Biswal BM, Zakaria A, Ahmad NM. Topical application of honey in the management of radiation mucositis: a preliminary study. Support Care Cancer 2003;11(4):242-248. doi:10.1007/s00520-003-0443-y.

[25] Hawley P, Hovan A, McGahan CE, Saunders D. A randomized placebocontrolled trial of manuka honey for radiation-induced oral mucositis. Support Care Cancer 2014;22(3):751-761. doi:10.1007/s00520013-2031-0. 
[26] Howlader D, Singh V, Mohammad S, Gupta S, Pal U, Pal M. Effect of topical application of pure honey in chemo-radiation-induced mucositis and its clinical benefits in improving quality of life in patients of oral squamous cell carcinoma. J Maxillofac Oral Surg 2019;18(1):7379. doi:10.1007/s12663-017-1077-9.

[27] Jayachandran S, Balaji N. Evaluating the effectiveness of topical application of natural honey and benzydamine hydrochloride in the management of radiation mucositis. Indian J Palliat Care 2012;18(3):190195. doi:10.4103/0973-1075.105689.

[28] Jayalekshmi JL, Lakshmi R, Mukerji A. Honey on oral mucositis: a randomized controlled trial. Gulf J Oncolog 2016;1(20):30-37.

[29] Khanal B, Baliga M, Uppal N. Effect of topical honey on limitation of radiation-induced oral mucositis: an intervention study. Int J Oral Maxillofac Surg 2010;39(12):1181-1185. doi:10.1016/j. ijom.2010.05.014.

[30] Maiti P, Ray A, Mitra TN, Jana U, Bhattacharya J, Ganguly S. The effect of honey on mucositis induced by chemoradiation in head and neck cancer. J Indian Med Assoc 2012;110(7):453-456.

[31] Motallebnejad M, Akram S, Moghadamnia A, Moulana Z, Omid $S$. The effect of topical application of pure honey on radiation-induced mucositis: a randomized clinical trial. J Contemp Dent Pract 2008;9(3):40-47. doi:10.5005/jcdp-9-3-40.

[32] Rashad UM, Al-Gezawy SM, El-Gezawy E, Azzaz AN. Honey as topical prophylaxis against radiochemotherapy-induced mucositis in head and neck cancer. J Laryngol Otol 2009;123(2):223-228. doi:10.1017/ S0022215108002478.

[33] Ceylan G, Yılmaz N, Nisbet HÖ, Nisbet C, Dede DÖ, Hoşgör F, et al. Honey in wound care in complete denture wearers: a pilot study. Mater Res Innov 2010;14(3):268-270. doi:10.1179/14330751 0X12719005364945.

[34] Halim DS, Mahanani ES, Saini R, Omar M, bt Ibrahi NR, Alam MK. A comparison study on the effectiveness of local honey and salicylate gel for treatment of minor recurrent aphtous stomatitis. Int Med J 2013;20(6):770-772.

[35] Al-Khanati NM, Al-Moudallal Y. Effect of intrasocket application of manuka honey on postsurgical pain of impacted mandibular third molars surgery: split-mouth randomized controlled trial. J Maxillofac Oral Surg 2019;18(1):147-152. doi:10.1007/s12663-018-1142-z.

[36] Lim YS, Kwon SK, Park JH, Cho CG, Park SW, Kim WK. Enhanced mucosal healing with curcumin in animal oral ulcer model. Laryngoscope 2016;126(2):E68-73. doi:10.1002/lary.25649.

[37] Sonis ST. Pathobiology of oral mucositis: novel insights and opportunities. J Support Oncol 2007;5(9 Suppl 4):3-11.

[38] Babaee N, Hosseinkazemi H, Pouramir M, Khakbaz Baboli O, Salehi $\mathrm{M}$, Khadir $\mathrm{F}$, et al. Salivary oxidant/antioxidant status and hematological parameters in patients with recurrent aphthous stomatitis. Caspian J Intern Med 2016;7(1):13-18.

[39] Bardy J, Molassiotis A, Ryder WD, Mais K, Sykes A, Yap B, et al. A double-blind, placebo-controlled, randomised trial of active manuka honey and standard oral care for radiation-induced oral mucositis. Br J Oral Maxillofac Surg 2012;50(3):221-226. doi:10.1016/j. bjoms.2011.03.005.

[40] Parsons E, Begley A, Herst P. Manuka honey mouthwash does not affect oral mucositis in head and neck cancer patients in New Zealand. J Radiother Pract 2012;11(4):249-256. doi:10.1017/ S1460396911000410.

[41] Jain A, Bhaskar DJ, Gupta D, Agali C, Gupta V, Gupta RK, et al. Comparative evaluation of honey, chlorhexidine gluconate $(0.2 \%)$ and combination of xylitol and chlorhexidine mouthwash $(0.2 \%)$ on the clinical level of dental plaque: a 30 days randomized control trial. Perspect Clin Res 2015;6(1):53-57. doi:10.4103/2229-3485.148819.

[42] Singhal R, Siddibhavi M, Sankeshwari R, Patil P, Jalihal S, Ankola A. Effectiveness of three mouthwashes - Manuka honey, Raw honey, and Chlorhexidine on plaque and gingival scores of 12-15-year-old schoo children: a randomized controlled field trial. J Indian Soc Periodontol 2018;22(1):34-39.

[43] Mavric E, Wittmann S, Barth G, Henle T. Identification and quantification of methylglyoxal as the dominant antibacterial constituent of Manuka (Leptospermum scoparium) honeys from New Zealand. Mol Nutr Food Res 2008;52(4):483-489. doi:10.1002/mnfr.200700282.

[44] Deng J, Liu R, Lu Q, Hao P, Xu A, Zhang J, et al. Biochemical properties, antibacterial and cellular antioxidant activities of buckwheat honey in comparison to manuka honey. Food Chem 2018;252:243-249. doi:10.1016/j.foodchem.2018.01.115.

[45] Allaman I, Bélanger M, Magistretti PJ. Methylglyoxal, the dark side of glycolysis. Front Neurosci 2015;9:23. doi:10.3389/fnins.2015.00023.

[46] Meeprom A, Sompong W, Suantawee T, Thilavech T, Chan CB, Adisakwattana $S$. Isoferulic acid prevents methylglyoxal-induced protein glycation and DNA damage by free radical scavenging activity. BMC Complement Altern Med 2015;15:346. doi:10.1186/s12906-0150874-2.

[47] Hossain MA, Piyatida P, da Silva JAT, Fujita M. Molecular mechanism of heavy metal toxicity and tolerance in plants: central role of glutathione in detoxification of reactive oxygen species and methylglyoxal and in heavy metal chelation. J Bot 2012;2012:872875 doi:10.1155/2012/872875

[48] Kurahashi T, Fujii J. Roles of antioxidative enzymes in wound healing. J Dev Biol 2015;3(2):57-70. doi:10.3390/jdb3020057.

[49] Choi CH, Park SJ, Jeong SY, Yim HS, Kang SO. Methylglyoxal accumulation by glutathione depletion leads to cell cycle arrest in Dictyos telium. Mol Microbiol 2008;70(5):1293-1304. doi:10.1111/j.13652958.2008.06497.x.

[50] Almasaudi SB, El-Shitany NA, Abbas AT, Abdel-dayem UA, Ali SS, Al Jaouni SK, et al. Antioxidant, anti-inflammatory, and antiulcer potential of manuka honey against gastric ulcer in rats. Oxid Med Cell Longev 2016;2016:3643824. doi:10.1155/2016/3643824. 